\title{
NONCOMMUTATIVE PROJ AND COHERENT ALGEBRAS
}

\author{
A. PolishchuK
}

\begin{abstract}
We prove that an abelian category equipped with an ample sequence of objects is equivalent to the quotient of the category of coherent modules over the corresponding algebra by the subcategory of finite-dimensional modules. In the Noetherian case a similar result was proved by Artin and Zhang in [2].
\end{abstract}

\section{INTRODUCTION}

The main result of this paper is a slight generalization of the theorem of Artin and Zhang in [2] characterizing certain class of abelian categories that can be viewed as noncommutative analogues of categories of coherent sheaves on projective schemes. Recall that the main idea of this approach to noncommutative projective geometry is to associate to a noncommutative graded algebra $A$ the quotient category QGR $A$ of the category of graded $A$-modules by the subcategory of torsion modules. If $A$ is commutative and is generated by a finite number of elements of degree 1 then by the theorem of Serre [10] the category of quasicoherent sheaves on $\operatorname{Proj}(A)$ is equivalent to QGR $A$. Therefore, one would like to think about QGR $A$ as a suitable replacement for the latter category in the case when $A$ is noncommutative. Theorem 4.5 of [2] gives a nice criterion for a locally Noetherian abelian category $\mathcal{C}$ to be equivalent to QGR $A$ for some $A$. Namely, the criterion says that $\mathcal{C}$ should contain a sequence of objects $\left(E_{n}, n \in \mathbb{Z}\right)$ satisfying some properties analogous to those of the sequence $\left(\mathcal{O}_{X}(n), n \in \mathbb{Z}\right)$ for a projective scheme $X$. We will refer to this criterion as the AZ-theorem and to $\left(E_{n}\right)$ as an ample sequence.

In the later developments of the above point of view on noncommutative geometry the assumption that the categories under consideration have to be (at least, locally) Noetherian was widely accepted as a convenient technicality. However, it appears that the list of categories considered by "noncommutative algebraic geometry" should also contain some non-Noetherian categories. One family of such examples is provided by the categories of holomorphic bundles on noncommutative tori. These categories can also be viewed as hearts of certain non-standard $t$-structures in derived categories of coherent sheaves on elliptic curves (see [8]). It is very easy to see that none of these categories is Noetherian. More precisely, every non-zero object in these categories is non-Noetherian. On the other hand, in the case when a noncommutative two-torus corresponds to a quadratic irrationality (has "real multiplication") there is a natural graded

Received January 17, 2003.

This work was partially supported by NSF grant DMS-0070967. 
algebra associated with this category and one expects to have an analogue of Serre's theorem. The goal of the present paper is to prove that a (not necessarily Noetherian) abelian category equipped with an ample sequence of objects can still be described in terms of the corresponding graded algebra. Applications to noncommutative two-tori are considered in [9].

We exploit the idea going back to Serre's paper [10] that the correct abelian category replacing the category of finitely generated modules in the non-Noetherian case is the category of coherent modules. Recall that an $A$-module $M$ is called coherent if $M$ is finitely generated and the kernel of every surjection $A^{\oplus n} \rightarrow M$ is finitely generated. An algebra $A$ is called coherent if it is coherent as a module over itself. The graded version of this definition can be reformulated as follows: a finitely generated graded algebra $A$ is coherent iff the module of relations between every finite collection of homogeneous elements in $A$ is finitely generated. Roughly speaking, our main result is that if one removes the assumption that the category is Noetherian in the AZ-theorem then the corresponding graded algebra is still coherent and the abelian category in question is equivalent to the quotient of the category of coherent modules by the subcategory of finitedimensional modules.

We did not study the question which coherent algebras appear in this way. Recall that in the Noetherian case the answer is formulated in terms of a rather unpleasant cohomological condition called $\chi_{1}$ (in the second part of the AZtheorem). It is not difficult to formulate a similar condition for coherent algebras (replacing finitely generated modules by coherent modules in the definition of [2]) and we expect that an obvious extension of the second part of the AZ-theorem is true in our situation.

One technical point: it is convenient to extend the class of graded algebras to the wider class consisting of $\mathbb{Z}$-algebras. These are associative algebras of the form $A=\oplus_{(i, j) \in \mathbb{Z}^{2}, i \leq j} A_{i j}$ such that the only non-zero products in $A$ are $A_{j k} \otimes A_{i j} \rightarrow A_{i k}$. The category of (nonnegatively) graded algebras is a subcategory of the category of $\mathbb{Z}$-algebras: to every graded algebra $A=\oplus_{n \geq 0} A_{n}$ one can associate a $\mathbb{Z}$-algebra $A_{\mathbb{Z}}=\oplus A_{i j}$ with $A_{i j}=A_{j-i}$. As was observed in [11], sec. 11.1, the AZ-theorem can be extended to the case when an ample sequence of objects does not have the form $\left(\sigma^{n}(O), n \in \mathbb{Z}\right)$ for some object $O$ and some autoequivalence $\sigma$, by working with $\mathbb{Z}$-algebras. Our generalization is also formulated using the language of $\mathbb{Z}$-algebras.

It is worth mentioning that the notion of a coherent $\mathbb{Z}$-algebra arises naturally in the theory of geometric helices developed in [4]. Namely, it was proved in [7] that in the situation when a triangulated category is generated by the geometric helix, certain natural pair of subcategories defines a $t$-structure iff the $\mathbb{Z}$-algebra associated to this helix is coherent.

It would be very desirable to develop some other techniques for checking whether a given graded algebra is coherent. We present some partial results in this direction which allow us to construct various examples of coherent and noncoherent algebras. Also, as a consequence of our main result we derive the 
connection between coherency of a graded algebra and its Veronese subalgebras. We believe that in the noncommutative world many natural constructions lead to coherent algebras (but not necessarily Noetherian ones). For example, Piontkovskii in [5] proved coherence of a graded algebra with finite number of generators and a finite number of defining monomial relations. In [6] this result is generalized to a broader class of algebras.

Acknowledgments. This note is mostly based on a part of the author's diploma work [7] carried out at Moscow State University in 1993 under the supervision of A. Bondal to whom I am very grateful. I also thank L. Positselski and D. Piontkovskii for helpful discussions.

Conventions. Throughout this paper we work over a fixed field $k$. By a graded algebra we mean a nonnegatively graded $k$-algebra of the form $A=\oplus_{i \geq 0} A_{i}$, where $A_{0}=k$ (thus, we consider only connected algebras).

\section{Preliminaries on $\mathbb{Z}$-algebras}

In this section we review some basic constructions dealing with $\mathbb{Z}$-algebras.

Definition.(see [3],[4]) A $\mathbb{Z}$-algebra is an associative $k$-algebra $A$ of the form $A=$ $\oplus_{i \leq j} A_{i j}, i, j \in \mathbb{Z}$, where $A_{i i}=k$ for all $i \in \mathbb{Z}$; the only non-zero components of multiplication with respect to this decomposition are $A_{j k} \otimes A_{i j} \rightarrow A_{i k}$; moreover, the multiplications $A_{j j} \otimes A_{i j} \rightarrow A_{i j}$ and $A_{i j} \otimes A_{i i} \rightarrow A_{i j}$ are the identity maps.

We will always impose the following finiteness condition on a $\mathbb{Z}$-algebra: $\operatorname{dim}_{k} A_{i j}<\infty$ for all $i \leq j$. We consider the category GR $A$ of graded right $A$-modules $M=\oplus_{i \in \mathbb{Z}} M_{i}$ with an $A$-action of the form $M_{j} \otimes A_{i j} \rightarrow M_{i}$, such that $M_{i} \otimes A_{i i} \rightarrow M_{i}$ are the identity maps. The morphisms in GR $A$ are homomorphisms of $A$-modules (they preserve the grading automatically). We denote by $S_{j}$ the unique irreducible $A$-module such that $\left(S_{j}\right)_{i}=0$ for $i \neq j,\left(S_{j}\right)_{j}=k$ and by $P_{j}=\oplus_{i} A_{i j}$ its indecomposable projective cover. Let $\mathcal{P}$ be the family of modules consisting of the finite direct sums of $P_{j}$ 's. We say that an $A$-module $M$ is finitely generated (resp., finitely presented) if there is a surjection $P \rightarrow M$ with $P \in \mathcal{P}$ (resp., $M=\operatorname{coker}\left(P^{\prime} \rightarrow P\right)$, where $\left.P, P^{\prime} \in \mathcal{P}\right)$. Note that our assumption on $A$ implies that for every finitely generated $A$-module $M=\oplus_{i} M_{i}$ the graded components $M_{i}$ are finite-dimensional.

As we already mentioned before, to a graded algebra $A=\oplus_{i \geq 0} A_{i}$ with $\operatorname{dim}_{k} A_{i}<\infty$ one can associate the $\mathbb{Z}$-algebra $A_{\mathbb{Z}}=\oplus A_{i j}$ with $A_{i j}=\bar{A}_{j-i}$. Note that the categories of graded right modules over $A$ and $A_{\mathbb{Z}}$ are equivalent: the equivalence sends an $A$-module $M=\oplus M_{i}$ to an $A_{\mathbb{Z}}$-module $M_{\mathbb{Z}}=\oplus M_{-i}$. Under this equivalence $P_{j}$ corresponds to the free module $A(j)$, where $A(j)_{i}=A_{i+j}$.

Let $A$ be a $\mathbb{Z}$-algebra. Henceforward, by an $A$-module we always mean a graded right $A$-module.

Definition. An $A$-module $M$ is called coherent if it satisfies the following two conditions: 
(i) $M$ is finitely generated;

(ii) for every homomorphism $f: P \rightarrow M$ with $P \in \mathcal{P}$ the module $\operatorname{ker}(f)$ is finitely generated.

Of course, this definition is essentially a particular case of the general definition of coherent sheaves of modules given by Serre in [10]. We denote by $\operatorname{coh} A \subset \mathrm{GR} A$ the full subcategory of coherent modules. Some basic properties of coherent modules proved in [10] also hold in our situation with same proofs. Most notably, we have the following result.

Proposition 1.1. coh $A$ is an abelian subcategory of GR $A$ closed under extensions.

Definition. A $\mathbb{Z}$-algebra $A$ is called weakly right coherent if all the modules $P_{j}$ are coherent. It is called right coherent if in addition all the modules $S_{j}$ are coherent.

Similarly, one defines the notion of a left coherent $\mathbb{Z}$-algebra. In this section and in the next one we work exclusively with right modules, so by coherence we mean right coherence. Considering graded algebras as a subcategory in the category of $\mathbb{Z}$-algebras we get a notion of coherence for them. Note that for a finitely generated graded algebra weak coherence and coherence are equivalent. Later we will prove that the tensor algebra $T(V)$ of a finite-dimensional space $V$ is coherent (see Corollary 3.2). This easily implies that the tensor algebra $T(V)$ of an infinite-dimensional vector space is weakly coherent (but not coherent).

Lemma 1.2. Assume that $A$ is weakly coherent. Then every coherent module $M$ has a resolution $\ldots P^{-2} \rightarrow P^{-1} \rightarrow P^{0} \rightarrow M$ with $P^{i} \in \mathcal{P}$.

Proof. Indeed, since $M$ is finitely generated, we can choose a surjection $f: P^{0} \rightarrow$ $M$, where $P^{0} \in \mathcal{P}$. By Proposition $1.1 \operatorname{ker}(f)$ is again coherent, so we can iterate this procedure.

One can easily prove the following criterion: a $\mathbb{Z}$-algebra $A$ is weakly coherent (resp., coherent) iff there exists a full abelian subcategory $\mathcal{C} \subset$ GR $A$ consisting of finitely generated modules, such that $\mathcal{P} \subset \mathcal{C}$ (resp., $\mathcal{P} \subset \mathcal{C}$ and $S_{j} \in \mathcal{C}$ for every $j$ ). This criterion can be used to prove the following result.

Proposition 1.3. Let $A \rightarrow B$ be a homomorphism of $\mathbb{Z}$-algebras and $\left\{Q_{j}, j \in\right.$ $\mathbb{Z}\}$ be the set of indecomposable projective $B$-modules (defined in the same way as $P_{j}$ for $\left.A\right)$. Assume that $A$ is coherent and all $Q_{j}$ are coherent as $A$-modules. Then $B$ is a coherent $\mathbb{Z}$-algebra.

Proof. This follows immediately from the above criterion applied to the subcategory of $B$-modules that are coherent over $A$.

For example, the above proposition implies that the quotient $A / J$ of a coherent graded algebra $A$ by a two-sided (homogeneous) ideal $J$, such that $J$ is finitely generated as a right ideal, is again a coherent algebra. 
A module $M=\oplus_{i} M_{i}$ over a $\mathbb{Z}$-algebra $A$ is called bounded (resp., bounded above) if $M_{i} \neq 0$ for only a finite number of indices $i$ (resp., $M_{i}=0$ for all sufficiently large $i$ ). Note that every finitely generated $A$-module is bounded above (since this is true for $P_{j}$ ). For an $A$-module $M=\oplus_{i} M_{i}$ and an integer $n \in \mathbb{Z}$ we denote by $M_{\leq n}$ the $A$-module $\oplus_{i \leq n} M_{i}$. For a coherent $\mathbb{Z}$-algebra $A$ we denote by $\operatorname{coh}^{b} A$ the category of bounded coherent modules. Note that since all the modules $S_{j}$ are coherent, $\operatorname{coh}^{b} A$ consists exactly of all finite-dimensional modules. Furthermore, $\operatorname{coh}^{b} A$ is a Serre subcategory of coh $A$, so the quotient category

$$
\operatorname{cohproj} A:=\operatorname{coh} A / \operatorname{coh}^{b}(A)
$$

is still abelian. In particular, we can construct such a category (still denoted $\operatorname{cohproj} A$ ) for a coherent graded algebra $A$. If $A$ is Noetherian then $\operatorname{cohproj} A$ coincides with the category qgr $A$ considered in [2].

\section{Coherent sequences and an equivalence of categories}

Let $\mathcal{C}$ be an abelian $k$-linear category, $\mathcal{E}=\left(E_{i}, i \in \mathbb{Z}\right)$ be a sequence of objects of $\mathcal{C}$. We are going to show that under appropriate conditions on $\mathcal{E}$ (that are combined below in the notion of a coherent sequence) certain quotient of the category $\mathcal{C}$ is equivalent to $\operatorname{cohproj} A$, where the $\mathbb{Z}$-algebra $A=A(\mathcal{E})=\oplus_{i \leq j} A_{i j}$ is defined as follows: $A_{i j}=\operatorname{Hom}_{\mathcal{C}}\left(E_{i}, E_{j}\right)$ for $i<j, A_{i i}=k$, the multiplication is induced by the composition in $\mathcal{C}$. In the particular case of an ample sequence, we will obtain an equivalence of $\mathcal{C}$ with $\operatorname{cohproj} A$.

Using this technique we will show that for a coherent graded algebra $A$ generated by $A_{1}$ over $A_{0}=k$ there is an equivalence of categories $\operatorname{cohproj} A \simeq$ cohproj $A^{(n)}$, where $A^{(n)}=\oplus_{i \geq 0} A_{i n}$ is a Veronese subalgebra of $A$. The similar result in the Noetherian case is due to Verevkin [12], Theorem (A-5) (see also [2], Prop. 5.10).

From now on we will always assume that the sequence $\mathcal{E}=\left(E_{i}\right)$ satisfies the following finiteness condition: for every object $X \in \mathcal{C}$ one has $\operatorname{dim}_{k} \operatorname{Hom}_{\mathcal{C}}\left(E_{i}, X\right)$ $<\infty$. In particular, the components $A_{i j}$ of the corresponding $\mathbb{Z}$-algebra $A$ are finite-dimensional.

Definition. A sequence $\mathcal{E}=\left(E_{i}\right)$ is called projective if for every surjection $X \rightarrow Y$ in $\mathcal{C}$ there exists $n \in \mathbb{Z}$ such that the corresponding map $\operatorname{Hom}\left(E_{i}, X\right) \rightarrow$ $\operatorname{Hom}\left(E_{i}, Y\right)$ is surjective for $i<n$.

Every sequence $\mathcal{E}=\left(E_{i}\right)$ defines a functor $\Gamma_{*}: \mathcal{C} \rightarrow \operatorname{GR} A$ sending $X$ to the right $A$-module $\oplus_{i \in \mathbb{Z}} \operatorname{Hom}\left(E_{i}, X\right)$. More important for us are truncated versions of this functor

$$
\Gamma_{\leq m} X:=\left(\Gamma_{*} X\right)_{\leq m}=\oplus_{i \leq m} \operatorname{Hom}\left(E_{i}, X\right),
$$

where $m \in \mathbb{Z}$. If $\mathcal{E}$ is projective then $\Gamma_{*}$ (resp., $\Gamma_{\leq m}$ ) is exact modulo the subcategory of bounded below (resp., bounded) modules. We define the subcategory $\mathcal{C}_{0}=\mathcal{C}_{0}(\mathcal{E}) \subset \mathcal{C}$ as the full subcategory consisting of objects $X$ such that $\operatorname{Hom}\left(E_{i}, X\right)=0$ for $i<<0$. If $\mathcal{E}$ is projective then $\mathcal{C}_{0}$ is a Serre subcategory. 
Definition. (i) A projective sequence $\mathcal{E}=\left(E_{i}\right)$ is called coherent if for every object $X \in \mathcal{C}$ and every $m \in \mathbb{Z}$ there exists a set of integers $i_{1}, \ldots, i_{s}$ with $i_{j} \leq m$ for all $j$, such that the canonical map

$$
\oplus_{j=1}^{s} \operatorname{Hom}\left(E_{i_{j}}, X\right) \otimes \operatorname{Hom}\left(E_{i}, E_{i_{j}}\right) \rightarrow \operatorname{Hom}\left(E_{i}, X\right)
$$

is surjective for $i<<0$.

(ii) A coherent sequence $\mathcal{E}=\left(E_{i}\right)$ is called ample if $\mathcal{C}_{0}(\mathcal{E})=0$.

The following lemma gives convenient reformulations of these conditions.

Lemma 2.1. Let $\mathcal{E}=\left(E_{i}\right)$ be a projective sequence.

(i) $\mathcal{E}$ is coherent iff for every object $X \in \mathcal{C}$ the $A$-modules $\Gamma_{\leq m} X$ are finitely generated for all $m$.

(ii) $\mathcal{E}$ is ample iff for every $X \in \mathcal{C}$ and every $m \in \mathbb{Z}$ there exists a surjection $\oplus_{j=1}^{s} E_{i_{j}} \rightarrow X$ for some (possibly repeating) indices $i_{1}, \ldots, i_{s}$ with $i_{j} \leq m$ for all $j$.

Proof. (i) Assume first that $\mathcal{E}$ is coherent. Note that for all $i \leq m$ the module $\Gamma_{\leq m} E_{i}$ contains $P_{i}$ as a submodule and the quotient is finite-dimensional, hence, $\Gamma_{\leq m} E_{i}$ is finitely generated in this case. Now for an object $X \in \mathcal{C}$ and an integer $m$ we choose integers $i_{1}, \ldots, i_{s} \leq m$ as in the definition and consider the morphism

$$
f: \oplus_{j} \operatorname{Hom}\left(E_{i_{j}}, X\right) \otimes E_{i_{j}} \rightarrow X,
$$

such that coker $\Gamma_{*} f$ is bounded below. Since by our assumption on $\mathcal{E}$ all spaces $\operatorname{Hom}\left(E_{i}, X\right)$ are finite-dimensional, this implies that coker $\Gamma_{\leq m} f$ is finitedimensional. On the other hand, as we observed above, the $A$-modules $\Gamma_{\leq m} E_{i_{j}}$ are finitely generated. It follows that $\Gamma_{\leq m} X$ is finitely generated. Conversely, if $\Gamma_{\leq m} X$ is finitely generated then there exists a surjection $\oplus_{j=1}^{s} P_{i_{j}} \rightarrow \Gamma_{\leq m} X$, where $i_{j} \leq m$ for all $j$, hence, the condition in the definition of coherence is satisfied for $m$.

(ii) Assume that $\mathcal{E}$ is ample. Then for every $m$ we can choose integers $i_{1}, \ldots, i_{s} \leq$ $m$ as in the definition of coherence and consider the morphism (2.1). We claim that coker $f=0$. Indeed, since $\mathcal{E}$ is projective, we have $\Gamma_{\leq n}(\operatorname{coker} f) \simeq$ coker $\Gamma_{\leq n} f=0$ for $n<<0$. Hence, coker $f$ belongs to $\mathcal{C}_{0}=0$. Conversely, assume that for every $X \in \mathcal{C}$ and $m \in \mathbb{Z}$ there exists a surjection $f: \oplus_{j=1}^{s} E_{i_{j}} \rightarrow X$, where $i_{j} \leq m$ for all $j$. Then by projectivity of $\mathcal{E}$ the map $\Gamma_{\leq n} f$ is surjective for $n<<0$ which implies that the sequence $\mathcal{E}$ is coherent. On the other hand, it is clear that in this case $\mathcal{C}_{0}=0$.

Corollary 2.2. If $A$ is a coherent $\mathbb{Z}$-algebra then the sequence $\left(P_{i}\right)$ in $\operatorname{coh} A$ is coherent.

Proof. Indeed, all truncations of a coherent module are still coherent. 
Remark. The above lemma shows that our definition of ampleness is a direct generalization of the definition (4.2.1) of [2].

Proposition 2.3. Let $\mathcal{E}=\left(E_{i}\right)$ be a coherent sequence, $A=A(\mathcal{E})$ be the corresponding $\mathbb{Z}$-algebra. Then

(i) for every $X \in \mathcal{C}$ and every $m \in \mathbb{Z}$ the $A$-module $\Gamma_{\leq m} X$ is coherent;

(ii) the $\mathbb{Z}$-algebra $A$ is coherent.

Proof. (i) From Lemma 2.1(i) we know that the modules $\Gamma_{\leq m} X$ are finitely generated. Let $f: \oplus_{j=1}^{s} P_{i_{j}} \rightarrow \Gamma_{\leq m} X$ be any homomorpism of $A$-modules. We have to show that ker $f$ is finitely generated. Clearly we can assume that $i_{j} \leq m$ for all $j$. Then $f$ corresponds to a morphism $\phi: \oplus_{j} E_{i_{j}} \rightarrow X$ in $\mathcal{C}$. Let $n$ be an integer smaller than all $i_{j}$. We have

$$
(\operatorname{ker} f)_{\leq n}=\operatorname{ker} \Gamma_{\leq n} \phi \simeq \Gamma_{\leq n}(\operatorname{ker} \phi)
$$

so by Lemma 2.1, this module is finitely generated. Hence, ker $f$ is also finitely generated.

(ii) By part (i) the modules $\left(P_{j}\right)_{\leq j-1}=\Gamma_{\leq j-1} E_{j}$ and $\Gamma_{\leq j} E_{j}$ are coherent for every $j$. Therefore, the exact triple

$$
0 \rightarrow \Gamma_{\leq j-1} E_{j} \rightarrow \Gamma_{\leq j} E_{j} \rightarrow \operatorname{Hom}\left(E_{j}, E_{j}\right) \otimes S_{j} \rightarrow 0
$$

implies that $S_{j}$ is a coherent $A$-module. But $P_{j}$ is an extension of $S_{j}$ by $\left(P_{j}\right)_{\leq j-1}$, so it is also coherent.

Now we can prove our main theorem that generalizes the first part of Corollary 4.6 in $[2]$.

Theorem 2.4. Let $\mathcal{E}=\left(E_{i}\right)$ be a coherent sequence, $A=A(\mathcal{E})$ be the corresponding algebra. Then there is an equivalence of categories $\mathcal{C} / \mathcal{C}_{0} \simeq \operatorname{cohproj} A$. In particular, if $\mathcal{E}$ is ample then $\mathcal{C} \simeq \operatorname{cohproj} A$.

Proof. Recall that according to Proposition 2.3 the $\mathbb{Z}$-algebra $A$ is coherent and for every $X \in \mathcal{C}$ and every $m \in \mathbb{Z}$ the $A$-module $\Gamma_{\leq m} X$ is coherent. Now any of the functors $\Gamma_{\leq m}: \mathcal{C} \rightarrow \operatorname{coh} A$ induces an exact functor

$$
\Phi: \mathcal{C} / \mathcal{C}_{0} \rightarrow \operatorname{cohproj} A
$$

such that $\Phi(X)=\Gamma_{\leq m}(X) \bmod \operatorname{coh}^{b} A$. To construct a functor in an opposite direction let us first consider for every $M \in \operatorname{coh} A$ the functor

$$
h_{M}: \mathcal{C} \rightarrow \operatorname{Vect}_{k}: X \mapsto \operatorname{Hom}_{\mathrm{GR} A}\left(M, \Gamma_{*} X\right),
$$

where Vect $_{k}$ is the category of vector spaces over $k$. We claim that $h_{M}$ is representable. Indeed, for every $i \in \mathbb{Z}$ the functor $h_{P_{i}}$ is represented by $E_{i}$. Now every coherent module $M$ has a presentation in the form $M=\operatorname{coker}(P \rightarrow Q)$, where $P, Q \in \mathcal{P}$. We have an exact sequence of functors $0 \rightarrow h_{M} \rightarrow h_{Q} \rightarrow$ $h_{P}$, where the functors $h_{P}$ and $h_{Q}$ are representable. Therefore, $h_{M}$ is also representable. Let us denote by $\Gamma^{*} M$ the unique representing object for $h_{M}$. Note that the functor $\Gamma^{*}: \operatorname{coh} A \rightarrow \mathcal{C}$ is right exact. 
For every $M \in \operatorname{coh} A$ there is a natural homomorphism of $A$-modules $M \rightarrow$ $\Gamma_{\leq m} \Gamma^{*} M$, where $M$ is concentrated in degrees $\leq m$. We claim that the induced morphism in cohproj $A$ is an isomorphism. Indeed, clearly this is true for $M=$ $P_{i}$. Now using the fact that every coherent module is a quotient of a module from $\mathcal{P}$ one can easily prove the required statement by diagram chasing (one should check surjectivity first - see [1] (3.13)(i),(iii)).

It follows that $\Gamma^{*} M \in \mathcal{C}_{0}$ for $M \in \operatorname{coh}^{b} A$, so $\Gamma^{*}$ induces a functor

$$
\Psi: \operatorname{cohproj} A=\operatorname{coh} A / \operatorname{coh}^{b} A \rightarrow \mathcal{C} / \mathcal{C}_{0}
$$

such that $\Phi \circ \Psi \simeq$ Id. Furthermore, for every $X \in \mathcal{C}$ and $m \in \mathbb{Z}$ there is a canonical morphism $\Gamma^{*} \Gamma_{\leq m} X \rightarrow X$ induced by the embedding $\Gamma_{\leq m} X \rightarrow \Gamma_{*} X$ (considered as an element in $\left.\operatorname{Hom}\left(\Gamma_{\leq m} X, \Gamma_{*} X\right) \simeq \operatorname{Hom}\left(\Gamma^{*} \Gamma_{\leq m} X, X\right)\right)$. This gives a natural transformation of functors $\alpha: \Psi \circ \Phi \rightarrow$ Id. We claim that $\alpha$ is an isomorphism. Indeed, let us denote by $\mathcal{P}^{\prime}$ the collection of all objects of $\mathcal{C} / \mathcal{C}_{0}$ of the form $\oplus_{j=1}^{s} E_{i_{j}}$. One can easily check that $\alpha_{X}: \Psi(\Phi(X)) \rightarrow X$ is an isomorphism in $\mathcal{C} / \mathcal{C}_{0}$ for $X \in \mathcal{P}^{\prime}$. Now we claim that for every $X \in \mathcal{C} / \mathcal{C}_{0}$ there exists a surjection $f: P \rightarrow X$ in $\mathcal{C} / \mathcal{C}_{0}$ with $P \in \mathcal{P}^{\prime}$. Indeed, as we have seen in the proof of Lemma 2.1(ii), for every $X$ there exists a morphism of the form $f: \oplus_{j=1}^{s} E_{i_{j}} \rightarrow X$ in $\mathcal{C}$ with $\operatorname{coker}(f) \in \mathcal{C}_{0}$. But such a morphism induces a surjection in $\mathcal{C} / \mathcal{C}_{0}$. Therefore, every $X \in \mathcal{C} / \mathcal{C}_{0}$ can be represented in the form $\operatorname{coker}(P \rightarrow Q)$ with $P, Q \in \mathcal{P}^{\prime}$. Since $\Psi$ is right exact, this implies that $\alpha_{X}$ is an isomorphism for all $X \in \mathcal{C} / \mathcal{C}_{0}$.

Remarks. 1. It should be not difficult to prove that the algebra $A(\mathcal{E})$ corresponding to an ample sequence $\mathcal{E}$ satisfies an analogue of the condition $\chi_{1}$ of [2]. Conversely, this condition for a coherent algebra $A$ should imply that $\left(P_{i}\right)$ is a projective (and hence, ample) sequence in $\operatorname{cohproj} A$. We leave the details for the reader.

2. It is easy to adapt the above theorem to the framework of graded algebras. Namely, if the category $\mathcal{C}$ is equipped with an autoequivalence $\sigma$ such that $\sigma\left(E_{i}\right)=E_{i+1}$, then the corresponding $\mathbb{Z}$-algebra comes from a graded algebra.

3. It should be possible to weaken the assumption that all spaces $\operatorname{Hom}\left(E_{i}, X\right)$ are finite-dimensional in the same way as it is done in Theorem 4.5 of [2]. We leave for the reader to explore this.

In the remainder of the paper we leave the general context of $\mathbb{Z}$-algebras and work exclusively with graded algebras. Theorem 2.4 can be applied to derive the following result about Veronese subalgebras.

Proposition 2.5. Let $A=\oplus_{i \geq 0} A_{i}$ be a graded coherent algebra, $A^{(n)}=\oplus A_{\text {in }}$ be its Veronese subalgebra for some $n>0$. Assume that $A$ is generated by $A_{1}$ over $k$. Then $A^{(n)}$ is coherent and cohproj $A \simeq \operatorname{cohproj} A^{(n)}$.

Proof. The idea is to apply Theorem 2.4 to the sequence $(A(i n), i \in \mathbb{Z})$ in $\operatorname{coh} A$. First, we claim that this sequence is coherent. Indeed, it is enough to check that 
for every coherent $A$-module $M$ and every $m \in \mathbb{Z}$ the $A^{(n)}$-module $\oplus_{i \geq m} M_{i n}$ is finitely generated. Clearly, it is sufficient to check this for $M=A(j)$, in which case this follows from the assumption that $A$ is generated by $A_{1}$ over $k$. It remains to prove that if $M_{i n}=0$ for $i>>0$, where $M$ is a coherent $A$-module, then $M_{i}=0$ for $i>>0$. To this end we observe that every element $x \in M$ of such a module satisfies $x \cdot A_{i}=0$ for $i>>0$ (here we use the condition that $A$ is generated by $A_{1}$ ). Since $M$ is finitely generated it follows that $M$ is finite-dimensional.

Proposition 2.6. Let $A$ be a graded algebra generated by $A_{1}$ over $k$ with a finite number of defining relations. Then $A$ is coherent iff $A^{(n)}$ is coherent.

Proof. The "only if" part follows from Proposition 2.5. To prove the "if" part by Proposition 1.3 it suffices to verify that $A$ is finitely presented as a right $A^{(n)}$-module. Note that there is a direct sum decomposition

$$
A=\oplus_{m=0}^{n-1} P^{m}
$$

in the category of $A^{(n)}$-modules, where $P^{m}=\oplus_{i} A_{m+i n}$. Thus, it is enough to check that all the $A^{(n)}$-modules $P^{m}, m=0, \ldots, n-1$, are finitely presented. First, we observe that they are finitely generated. Indeed, the fact that $A$ is generated by $A_{1}$ immediately implies that the $A^{(n)}$-module $P^{m}$ is generated by $A_{m} \subset P^{m}$. Since there is a finite number of defining relations between generators of degree 1 , we have an exact sequence of $A$-modules

$$
\oplus_{i=2}^{d} V_{i} \otimes A(-i) \rightarrow A_{1} \otimes A(-1) \rightarrow A \rightarrow k \rightarrow 0,
$$

where $V_{i}$ are finite-dimensional vector spaces. Therefore, we have exact sequences of $A^{(n)}$-modules of the form

$$
\oplus_{i} V_{i} \otimes P^{m-i}\left(-a_{m, i}\right) \rightarrow A_{1} \otimes P^{m-1} \rightarrow P^{m} \rightarrow 0
$$

$m=1, \ldots, n-1\left(\right.$ where $\left.a_{m, i} \in \mathbb{Z}\right)$. Now we use a simple observation that for any homomorphism of modules $f: M_{1} \rightarrow M_{2}$, such that $M_{1}$ is finitely generated and $M_{2}$ is finitely presented, the module coker $f$ is finitely presented. Therefore, from the above sequences we can derive by induction in $m$ that $P^{m}$ is finitely presented for $0 \leq m \leq n-1$.

Remark. The condition that $A$ has a finite number of defining relations in Proposition 2.6 cannot be omitted. Indeed, let $A$ be the algebra generated by $x$ and $y$ with the defining relations $x^{2} y=0, y x^{2}=0, y x y=0, x y^{2 n+1} x=0$ for $n \geq 0$. Then $A$ is not coherent since it has an infinite number of relations. On the other hand, it is easy to see that the algebra $A^{(2)}$ has a finite number of monomial relations and hence coherent by the result of [5]. 


\section{Examples of coherent and noncoherent algebras}

Throughout this section $A$ denotes a finitely generated graded algebra.

We'll start with two reformulations of the coherence condition for such an algebra.

Proposition 3.1. The following conditions are equivalent:

(i) A is right coherent;

(ii) for every finitely generated right (homogeneous) ideal $J \subset A$ the space $\operatorname{Tor}_{1}^{A}(J, k)$ is finite-dimensional;

(iii) for every $M \in \mathrm{GR} A$ such that the spaces $\operatorname{Tor}_{0}^{A}(M, k)$ and $\operatorname{Tor}_{1}^{A}(M, k)$ are finite-dimensional, the space $\operatorname{Tor}_{2}^{A}(M, k)$ is also finite-dimensional.

Proof. Note that the condition $\operatorname{dim}_{k} \operatorname{Tor}_{0}^{A}(M, k)<\infty$ simply means that $M$ is finitely generated.

(iii) $\Longrightarrow$ (ii) This follows from the isomorphism $\operatorname{Tor}_{i}^{A}(J, k) \simeq \operatorname{Tor}_{i+1}^{A}(A / J, k)$ for all $i \geq 0$.

(ii) $\Longrightarrow$ (i) Since $A$ is finitely generated over $k$, it is enough to check that for every homomorphism $f: P \rightarrow A$, where $P \in \mathcal{P}$, the module $\operatorname{ker} f$ is finitely generated. Let $J$ be the image of $f$. Then $J$ is finitely generated, hence $\operatorname{Tor}_{1}^{A}(J, k)$ is finite-dimensional. Now from the exact sequence

$$
0 \rightarrow \operatorname{Tor}_{1}^{A}(J, k) \rightarrow \operatorname{Tor}_{0}^{A}(\operatorname{ker} f, k) \rightarrow \operatorname{Tor}_{0}^{A}(P, k) \rightarrow \ldots
$$

we derive that $\operatorname{Tor}_{0}^{A}(\operatorname{ker} f, k)$ is finite-dimensional. Hence, ker $f$ is finitely generated.

(i) $\Longrightarrow$ (iii) Let $M$ be a module with finite-dimensional $\operatorname{Tor}_{0}^{A}(M, k)$ and $\operatorname{Tor}_{1}^{A}(M, k)$. Then the minimal free resolution of $M$ has form

$$
\ldots \rightarrow P^{1} \rightarrow P^{0} \rightarrow M \rightarrow 0
$$

where $P^{0}, P^{1} \in \mathcal{P}$. It follows that $M$ is a coherent $A$-module, so it has resolution by modules in $\mathcal{P}$ (see Lemma 1.2). Hence $\operatorname{dim}_{k} \operatorname{Tor}_{i}^{A}(M, k)<\infty$ for all $i$.

Corollary 3.2. Let $V$ be a finite-dimensional vector space over $k$. Then the tensor algebra $T(V)$ is coherent.

Proof. Indeed, in this case $\operatorname{Tor}_{2}^{A}(M, k)=0$ for every $M$.

It is not difficult to see that a finitely generated $T(V)$-module is coherent if and only if its sufficiently far truncation is a free $T(V)$-module. This implies that every object of the category cohproj $T(V)$ is isomorphic to an object coming from $\mathcal{P}$. However, the morphism spaces in cohproj $T(V)$ between $P_{i}$ and $P_{j}$ are infinite-dimensional. An amusing observation is that for every $i \in \mathbb{Z}$ one has $P_{i} \simeq P_{i-1}^{\oplus \operatorname{dim} V}$ in cohproj $T(V)$.

The next two propositions provide some examples of coherent and noncoherent algebras. 
Proposition 3.3. Assume that $A$ has a decomposition $A=I \oplus B$, where $I$ is a homogeneous left ideal, $B$ is a graded subalgebra. Assume also that $B$ is a right Noetherian ring and that $I$ is free as a left $A$-module. Then $A$ is right coherent.

Proof. We are going to use criterion (iii) of Proposition 3.1. For every right $A$ module $M$ there is spectral sequence with $E_{p, q}^{2}=\operatorname{Tor}_{q}^{B}\left(\operatorname{Tor}_{p}^{A}(M, B), k\right)$ converging to $\operatorname{Tor}_{*}^{A}(M, k)$. On the other hand, the exact sequence $0 \rightarrow I \rightarrow A \rightarrow B \rightarrow 0$ of left $A$-modules shows that $\operatorname{Tor}_{p}^{A}(M, B)=0$ for $p \geq 2$. Now assume that $\operatorname{Tor}_{0}^{A}(M, k)$ and $\operatorname{Tor}_{1}^{A}(M, k)$ are finite-dimensional. Then

$$
\operatorname{dim}_{k} \operatorname{Tor}_{0}^{B}\left(\operatorname{Tor}_{0}^{A}(M, B), k\right)<\infty
$$

hence, $\operatorname{Tor}_{0}^{A}(M, B)$ is a finitely generated (right) $B$-module. This implies that all spaces $E_{0, q}^{2}$ are finite-dimensional. Therefore, from the assumption

$$
\operatorname{dim}_{k} \operatorname{Tor}_{1}^{A}(M, k)<\infty
$$

we derive that

$$
E_{1,0}^{2}=\operatorname{Tor}_{0}^{B}\left(\operatorname{Tor}_{1}^{A}(M, B), k\right)
$$

is finite-dimensional. Hence $\operatorname{Tor}_{1}^{A}(M, B)$ is a finitely generated $B$-module, which implies that all spaces $E_{1, q}^{2}$ are also finite-dimensional.

Corollary 3.4. Let $B=k \oplus B_{+}$be a graded algebra and set $A=B\langle z\rangle /\left(z B_{+}\right)$, where $B\langle z\rangle$ is the free product of $B$ with $k[z], \operatorname{deg}(z)=1$. If $B$ is right Noetherian then $A$ is right coherent. If $B$ is infinite-dimensional then $A$ is not right Noetherian.

Proof. To prove the first assertion apply the above proposition to the decomposition $A=A z \oplus B$. If $V_{1} \subset V_{2} \subset \ldots$ is a strictly decreasing sequence of vector subspaces in $B_{+}$then the right ideal $\oplus_{n \geq 1} V_{n} z^{n}$ in $A$ is infinitely generated.

Proposition 3.5. Let $B=\oplus_{i \geq 0} B_{i}$ be a graded algebra equipped with a decomposition $B=I \oplus C$, where $I$ is a homogeneous right ideal and $C$ is a graded subalgebra. Let us define the algebra $A$ as the quotient of the algebra $B\langle z\rangle$ (where $z$ has degree 1) by the relations $z I=0$ and $z c=c z$ for all $c \in C$. Assume that $I$ is not finitely generated as a right $B$-module. Then the algebra $A$ is not right coherent.

Proof. We have a decomposition $A=\oplus_{n \geq 0} B z^{n}$ and an exact sequence of left $A$-modules

$$
0 \rightarrow A \stackrel{i}{\rightarrow} A \rightarrow B \rightarrow 0
$$

where $i(x)=x \cdot z$. Let $J \subset A$ be the right ideal generated by $z$, so that $J=\oplus_{n \geq 1} C z^{n}$. By Proposition 3.1 it suffices to check that $\operatorname{dim}_{k} \operatorname{Tor}_{1}^{A}(J, k)=\infty$. Consider the spectral sequence with $E_{p, q}^{2}=\operatorname{Tor}_{q}^{B}\left(\operatorname{Tor}_{p}^{A}(J, B), k\right)$ converging to $\operatorname{Tor}_{*}^{A}(J, k)$. The above exact sequence shows that $\operatorname{Tor}_{i}^{A}(J, B) \simeq \operatorname{Tor}_{i+1}^{A}(A / J, B)=$ 
0 for $i \geq 1$, so this spectral sequence degenerates in the term $E_{2}$ and we obtain an isomorphism

$$
\operatorname{Tor}_{1}^{A}(J, k) \simeq \operatorname{Tor}_{1}^{B}\left(J \otimes_{A} B, k\right) \simeq \operatorname{Tor}_{1}^{B}(C, k) .
$$

But $C \simeq B / I$, so $\operatorname{Tor}_{1}^{B}(C, k) \simeq \operatorname{Tor}_{0}^{B}(I, k)$ which has infinite dimension.

Examples. 1. The algebra $A$ with generators $x, y, z$ and relations $x y=0, y z=$ $0, x z=z x$ is neither right nor left coherent. Indeed, this follows from Proposition 3.5 since we can represent $A$ in the form $B\langle x\rangle /(x I, x z-z x)$, where $B=k\langle y, z\rangle /(y z), I=B y B=\oplus_{n \geq 0} z^{n} y B$, and in the form $B^{\prime}\langle z\rangle /\left(I^{\prime} z, x z-z x\right)$, where $B^{\prime}=\langle x, y\rangle /(x y), I^{\prime}=B^{\prime} y \bar{B}^{\prime}=\oplus_{n \geq 0} B^{\prime} y x^{n}$.

2. The algebra $A$ with generators $x, y, z$ and relations $y z=0, x z=z x$ is not left coherent (since the two-sided ideal generated by $y$ in $k\langle x, y\rangle$ is infinitely generated as a left ideal). On the other hand, we claim that it is right coherent. Indeed, we can apply Proposition 3.3 to the decomposition $A=I \oplus k[x, z]$, where $I$ is the two-sided ideal generated by $y$. The fact that $I$ is a free left $A$-module follows from the decomposition $I=A y \oplus A y x \oplus A y x^{2} \oplus \ldots$

\section{References}

[1] M. Artin, M. Van der Bergh, Twisted homogeneous coordinate rings, J. Algebra 133 (1990), 249-271.

[2] M. Artin, J. J. Zhang, Noncommutative projective schemes, Advances in Math. 109 (1994), 228-287.

[3] A. Beilinson, V. Ginzburg, V. Schechtman, Koszul duality, J. Geom. Phys 5, 3 (1988), 317-350.

[4] A. Bondal, A. Polishchuk, Homological properties of associative algebras: method of helices, Russian Acad. Sci. Izv. Math. 42 (1994), 219-260.

[5] D. I. Piontkovskii, Gröbner bases and the coherence of monomial associative algebras, Fundam. Prikl. Mat. 2 (1996), 501-509.

[6] _ Noncommutative Gröbner bases, coherence of associative algebras, and divisibility in semigroups, Fundam. Prikl. Mat. 7 (2001), 495-513.

[7] A. Polishchuk, On coherent algebras, diploma work, Moscow State University (1993).

[8] A. Polishchuk, A. Schwarz, Categories of holomorphic vector bundles on noncommutative two-tori, Comm. Math. Phys. 236 (2003), 135-159.

[9] A. Polishchuk, Noncommutative two-tori with real multiplication as noncommutative projective varieties, J. Geom. Phys. 50 (2004), 162-187.

[10] J.-P. Serre, Faisceaux algébrique cohérents, Ann. of Math. 61 (1955), 197-278.

[11] J. T. Stafford, M. Van den Berg, Noncommutative curves and noncommutative surfaces, Bull. AMS 38 (2001), 171-216.

[12] A. B. Verevkin, On a non-commutative analogue of the category of coherent sheaves on a projective scheme, AMS Transl. 151 (1992), 41-53.

Department of Mathematics, University of Oregon, Eugene OR 97403

E-mail address: apolish@math.uoregon.edu 